\title{
Achieving Color Uniformity Across Multi-Projector Displays
}

\author{
Aditi Majumder \\ Zhu He \\ Herman Towles \\ Department of Computer Science, \\ University of North Carolina at Chapel Hill. \\ $\{$ majumder,hez,towles,welch\}@cs.unc.edu
}

Greg Welch

\begin{abstract}
Large area tiled displays are gaining popularity for use in collaborative immersive virtual environments and scientific visualization. While recent work has addressed the issues of geometric registration, rendering architectures, and human interfaces, there has been relatively little work on photometric calibration in general, and photometric non-uniformity in particular. For example, as a result of differences in the photometric characteristics of projectors, the color and intensity of a large area display varies from place to place. Further, the imagery typically appears brighter at the regions of overlap between adjacent projectors.

In this paper we analyze and classify the causes of photometric non-uniformity in a tiled display. We then propose a methodology for determining corrections designed to achieve uniformity, that can correct for the photometric variations across a tiled projector display in real time using per channel color look-up-tables (LUT).
\end{abstract}

CR Categories: 1.3.5 [Computer Graphics]: Projector Graphics and Optics

Keywords: Large Area Display, Tiled Displays, Projector Graphics, Color Calibration.

\section{Introduction}

Large area displays provide new and stimulating areas of research and have the potential to change the way humans interact with their computing environments. Large, high-resolution, tiled displays that can extend to the walls, ceilings and floors [4], have several advantages over traditional small computer screens. The high resolution and wide field-of-view (FOV) of such displays make them very useful for visualizing scientific data (PowerWall of University of Minnesota, tiled projector displays of Sandia National Laboratories and Argonne National Laboratories) and complex open environments like battlefields (Interactive DataWall of Rome Labs). They can be used as immersive surround displays (CAVE ${ }^{\mathrm{TM}}$ of University of Illinois at Chicago [2]) to create a compelling sense of presence and immersion in a virtual environment. The life-size objects enable a natural interaction between multiple humans and virtual objects making such displays a wonderful tool for collaborative workspaces
(Scalable Display Wall at Princeton [3], Interactive Mural at Stanford [1] and DataWall of MIT Media Lab).

There are several issues that are important when considering the development of large tiled display that is to operate as a single logical display.

1. Geometric Registration across Different Projectors: Recent years have seen a large amount of work done in this direction $[4,5,6,7]$, which provided most of the solutions for geometric registration, even for arbitrarily shaped displays using arbitrarily positioned projectors. Thus, we can now envision building more flexible tiled display systems, not being limited to planar displays or requiring rigid mechanical projector alignment.

2. Rendering Architecture and Algorithms: Interaction is likely to demand performance. While Infinity Wall or Power Wall are built using high-end multi-pipelined SGI graphics systems, more recent work has focussed on creating lower-cost, distributed PC rendering clusters.

3. Human Interface: The large tiled displays made up of multiple projectors, each of which may be driven by different graphics engines, should offer a human interface that hides this distributed nature of the system from a naive user and appears to be a single logical display. There has been some nice work in this direction at Stanford[1] and Princeton[3].

4. Photometric Uniformity: The projectors constituting the large area display, differ in their color/photometric characteristics. Sometimes projectors are found to have color non-uniformities even within their own field of view. Further, the overlap region between projectors appear brighter than the non-overlapping areas. These result in photometric non-uniformity across a large tiled multiprojector display.

This photometric non-uniformity is very distracting even when there is a perfect geometric registration and can break the illusion of a single display device. Several perceptual and psychological studies on human color perception and acuity support this observation [20]. These studies show that humans are able to easily perceive a $2 \%$ difference in brightness and a difference in color resulting from a change as small as $2 \mathrm{~nm}$ in the wavelength of light.

The comments in recent works on large tiled displays $[8,9,1$, $4,6]$, our interaction with others working in the same area, and our own experience has led us to believe that the problem of photometric non-uniformity of a multi-projector display is non-trivial and cannot be solved by some ad hoc means. We need a reliable method to calibrate the projectors photometrically which can be easily repeated periodically as the projectors change photometrically.

In this paper, we endeavor to develop a rigorous characterization and analysis of the problem and find practical means of solving it in a structured fashion. We use a spectroradiometer to accurately characterize the photometric properties of various projectors. Using this information and fundamental photometric principles we develop methods to match the photometric characteristics of different projectors using per channel color LUTs. 


\subsection{Main Contribution}

The following are the main contributions of this work.

1. Analysis and Classification : We analyze and classify the causes of photometric non-uniformity in a tiled display.

2. Photometric Calibration : We describe a methodology to calibrate/match the photometric characteristics of different projectors in real-time using independent per channel color maps. For this, we use an optical sensing device as a feedback device. The results are repeatable and do not depend on human intervention. Further, our solution is based on basic principles of photometry/colorimetry and achieves the best matching that is possible using independent color LUTS.

3. Practical Demonstration : We also demonstrate a way to implement the corrections given the capabilities of present hardware to achieve the photometric uniformity in real-time. Our real-time implementation utilizes RGB color look-up-table (LUT), available in all image generators, to make independent channel corrections.

In Section 2, we analyze the causes of photometric nonuniformity in multi-projector displays and identify parts of the problem that can be approached independently. In Section 3, we present the theories and definitions that will be used in the rest of the paper. In Section 4, we propose an algorithm to solve parts of the problem in real-time using a per channel color LUT. In Section 5, we demonstrate the implementation of our algorithm by matching the color of different projectors such that geometrically registered images from two or more projectors appear look photometrically constant. Then we present the results in Section 6 and finally conclude by giving some insight on the work that still needs to be done in this direction.

\section{Causes of Photometric Non-Uniformity in Multi-Projector Displays}

We categorize the problems of achieving photometric uniformity for a multi-projector display into four classes. Solving all of these problems will, in theory, achieve a photometrically uniform multiprojector display.

The problems can be classified as :

\section{Device Dependent}

These problems are completely dependent on the projector characteristics.

(a) Photometric Variation Between Different Projectors Two projectors have photometric variation due to differences in gamma, color gamut and the luminance range.

(b) Photometric Variation Within a Projector's FOV

i. A very regular effect that we have observed is the radial attenuation of brightness from the center to the edges of the projector's FOV.

ii. More irregular effects include the "blotchiness" of different hues in different regions of the projector's FOV.

\section{Application Dependent}

These problems are created by the way the projectors are used to form the multi-projector display.

(a) Photometric Variation Due to Overlapping Projectors The region of the display surface where multiple projectors overlap will look brighter than the other regions and, as a result, the image projected will have a 'seam'.

(b) Photometric Variation due to Non-Planar NonLambertian Display Surface i. The reflection of light from one region of the display surface to another is called color bleeding. One region of the display surface acts as a secondary light source for another region.

ii. In case of an arbitrarily curved non-diffuse surface, the amount of light reaching a point on the surface from the projector depends on the normal, the coefficient of reflectance at that point and the angle between the normal and the viewing direction.

The seams due to overlapping projectors in II.a above, have been addressed previously $[6,7]$ by employing a roll-off function such as a linear ramp or a cosine curve to smooth the intensity transition between projectors. The problem of $I I . b$ is complex and beyond the scope of this paper.

In this paper, we address the device dependent problems in $I$ above. Our goal is to change the input image to each projector in real-time in such a way that the large tiled display formed by the projection of these changed input images look photometrically seamless. To this end, we propose using the per channel color LUT available in all image generators and map the same input in different regions of the tiled display differently such that the projection of the mapped inputs are perceivably similar.

\section{Color Model}

We present here a brief overview of the color model we use. More elaborate discussion can be found in [22, 21, 20].

The human visual spectrum includes lights of wavelength in the range of $400-700 \mathrm{~nm}$. There are three types of color : achromatic (equal contribution from every wavelength) color which is perceived as gray, monochromatic (light of a single wavelength) which is impossible to generate physically and chromatic color (different contributions from different wavelengths). Hue is the sensation of color and differentiates a chromatic or monochromatic color from an achromatic color. A chromatic color with a broad spectrum is said to have low saturation, while a chromatic color with a narrow spectrum has a higher saturation. A pure monochromatic color has $100 \%$ saturation.

Several color specification systems have been developed to describe the different properties of color. These systems do not depend on any particular device, and hence are referred to as device independent color spaces.

CIEXYZ Color Space : This color space was established in 1931 by the CIE (Commission Internationale de Eclairage). In this color space, every color can be expressed as a linear combination of the CIE standard primaries, $\bar{X}, \bar{Y}$ and $\bar{Z}$.

$$
C=X \bar{X}+Y \bar{Y}+Z \bar{Z}
$$

where $C$ is the color, and $X, Y$ and $Z, 0 \leq X, Y, Z \leq 1$, are the CIE tristimulus values. $Y$ is called the luminance of $C$.

The chromaticity values $x, y$ and $z$ are defined from the tristimulus values as follows.

$$
x=\frac{X}{X+Y+Z} ; y=\frac{Y}{X+Y+Z} ; z=\frac{Z}{X+Y+Z}
$$

$(x, y)$ is called the chromaticity coordinates of a color and provides a measure of the chrominance (hue and saturation) of a color, while $Y$ gives the sense of brightness. Thus $(x, y, Y)$ uniquely defines any colored light in this CIEXYZ color space and is defined as the response of the colored light in this color space.

The cone shaped volume shown in Figure 1 contains the visible colors in the $\bar{X} \bar{Y} \bar{Z}$ color space. Now, let us consider the set of 


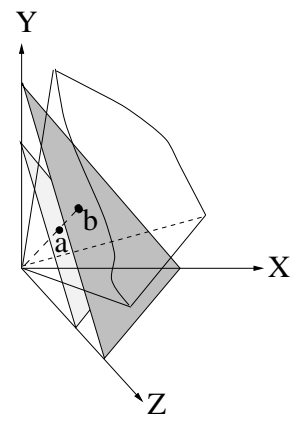

Figure 1: The cone of visible colors in the CIE color space

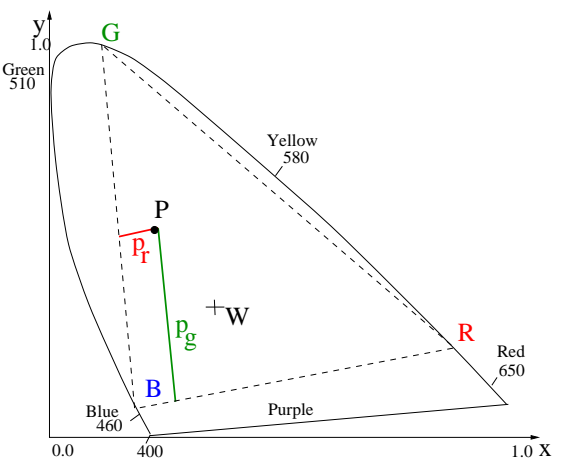

Figure 2: CIE Chromaticity Diagram

planes, $X+Y+Z=c$ where $c$ is a constant. Two such planes are shown by the shaded triangles in Figure 1. Let us take two colors $a$ and $b$ on the two different triangular planes but on the same straight line starting from the origin. These two colors have the same hue and saturation $((x, y))$ but different luminance $(Y)$.

Figure 2 shows the result of plotting $x$ and $y$ for all visible colors. This is called the CIE chromaticity diagram. The interior and the boundary of the horse-shoe shaped region represent all the visible chromaticity values. A standard white light is formally defined at $W$ where $x=y=z=\frac{1}{3}$. The $100 \%$ saturated monochromatic colors form the outer border of this region while the colors in the interior of the horse-shoe are desaturated. A straight line joining $W$ and any point on the border, represents the locus of the same hue with different levels of saturation. This diagram factors out the luminance and shows only the chrominance on constant luminance plane. When two colors are added together, the new color lies on the straight line joining the two original colors in the chromaticity diagram.

Chromaticity diagram helps to visualize the color gamuts. All the colors that can be reproduced by a device lies within the triangle joining the three color primaries $R, G$ and $B$ of a device in the chromaticity diagram. Thus, chromaticity diagram helps us compare the color primaries in different color devices, and needless to say, none of them can reproduce all the visible colors. The triangle in dotted lines in Figure 2 shows one such color gamut. If the chromaticity coordinates of $R, G$ and $B$ are $\left(R_{x}, R_{y}\right),\left(G_{x}, G_{y}\right)$ and $\left(B_{x}, B_{y}\right)$ respectively, then the chromaticity coordinates $\left(P_{x}, P_{y}\right)$ of a color $P=(r, g, b)$ with respect to the chromaticity of $R, G$ and $B$ is

$$
\begin{aligned}
& P_{x}=B_{x}+p_{r}\left(R_{x}-B_{x}\right)+p_{g}\left(G_{x}-B_{x}\right) \\
& P_{y}=B_{y}+p_{r}\left(R_{y}-B_{y}\right)+p_{g}\left(G_{y}-B_{y}\right)
\end{aligned}
$$

where $p_{r}=\frac{r}{r+g+b}, p_{g}=\frac{g}{r+g+b}$.
Distance Measures : The distance between two colors can be measured using different distance metric starting from the simple Euclidian distance to more complex perception based distance metric. For our work we use a measure similar to the perception based $E_{94}$ metric derived by Katoh [10]. Like $E_{94}$, our distance metric $D$ is dependent on three difference terms - one luminance and two chromaticity. For two colors, $a$ and $b$, where the responses of $a$ and $b$ are $R(a)=\left(Y_{a}, x_{a}, y_{a}\right)$ and $R(b)=\left(Y_{b}, x_{b}, y_{b}\right)$ respectively, the distance $D$ is defined as follows.

$$
D(R(a), R(b))=\sqrt{\left(\frac{Y_{a}-Y_{b}}{Y_{w}}\right)^{2}+\left(\frac{x_{a}-x_{b}}{S_{x}}\right)^{2}+\left(\frac{y_{a}-y_{b}{ }^{2}}{S_{y}}\right.}
$$

where $Y_{w}$ is a normalization factor, $S_{x}=1+\beta \sqrt{x_{a} x_{b}}, S_{y}=$ $1+\theta \sqrt{y_{a} y_{b}}$ with $\beta=0.045$ and $\theta=0.015$, as defined in $E_{94}$. For our purpose, we define $Y_{w}$ as the maximum luminance that can be generated by a projector. In practice, we consider the two colors matched if $D(R(a), R(b)) \leq \epsilon$ where $\epsilon$ is a small positive quantity.

\section{Algorithm Overview}

We present an algorithm which needs minimal human intervention. Our approach is to measure and match colors from different projectors against a standard, device independent color space. We use a Photo Research PR-705 spectroradiometer as our reference sensor.

\subsection{Problem Definition}

Let us assume that we are trying to achieve photometric uniformity across an $n$ projector display system with projectors $p, 0 \leq p<n$ with resolution of $w_{p} \times h_{p}$. Based on the analysis in Section 3, we can assume that the color projected by projector $p$ for input $i=$ $\left(i_{r}, i_{g}, i_{b}\right), 0.0 \leq i_{r}, i_{g}, i_{b} \leq 1.0$ at pixel $(s, t)$, is a function of $i$, $p, s$ and $t, P_{p}(i, s, t)$. The condition for photometric uniformity of $n$ projectors is, for any input $i$, the colors perceived at every pixel of every projector are equivalent. More formally, for any two pixel $(s, t)$ and $\left(s^{\prime}, t^{\prime}\right)$ of any two projector $p$ and $p^{\prime}$ respectively,

$$
\forall i, P_{p}(i, s, t) \equiv P_{p^{\prime}}\left(i, s^{\prime}, t^{\prime}\right)
$$

Of course in practice, Equation 4 is seldom true because of the device dependent problems outlined in Section 2.

\subsection{Our Approach - Color Mapping}

Our goal is to develop a color map so that the same input in all projector pixels produce perceptually identical input color. Now, let $I$ be the set of all inputs $i$. A color map is a function that maps each input color to a new input color. In order to achieve photometric uniformity, we need to generate color maps $C_{p s t}$ and $C_{p^{\prime} s^{\prime} t^{\prime}}$ for every pixel $(s, t)$ and $\left(s^{\prime}, t^{\prime}\right)$ of projector $p$ and $p^{\prime}$ respectively, such that $\forall s, t, s^{\prime}, t^{\prime}, p, p^{\prime}, i$,

$$
P_{p}\left(C_{p s t}(i), s, t\right) \equiv P_{p^{\prime}}\left(C_{p^{\prime} s^{\prime} t^{\prime}}(i), s^{\prime}, t^{\prime}\right)
$$

If Equation 5 is satisfied, we say that the color maps $C_{p s t}$ and $C_{p^{\prime} s^{\prime} t^{\prime}}^{\prime}$ have equivalent response and denoted it by

$$
C_{p s t}(i) \equiv C_{p^{\prime} s^{\prime} t^{\prime}}(i)
$$

Thus for the same input $l$, if we use the input specified by the color map at that pixel of that projector, then we can satisfy Equation 4 , and will have photometric uniformity across all projectors.

Given a 24-bit color representation, the task is to map each of the $2^{24}$ color inputs to a new input. Further, if we have to take care of the photometric variations within projector's own FOV, a large 

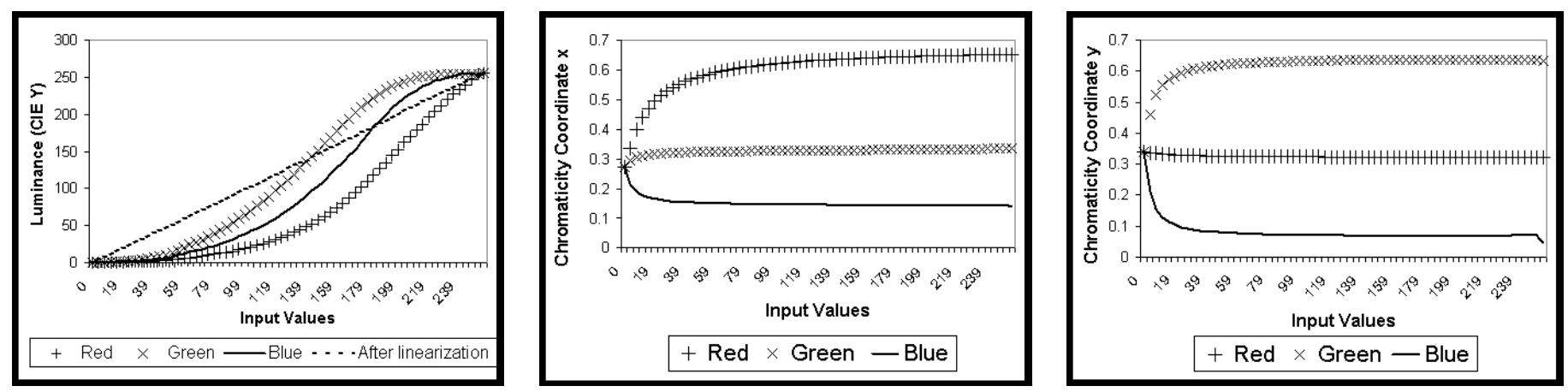

Figure 3: Left : Luminance Response before and after Non-Linearity Correction; Middle : Chromaticity $x$ is near constant with the increase in input values; Right : Chromaticity $y$ is near constant with the increase in input values

color map could conceivably be generated for every pixel $(s, t)$. Clearly, mapping every possible input for every pixel location is impractical today.

However, there are two ways to reduce the complexity of the problem. We can subsample in the spatial domain and use a smaller number of color maps (not for every pixel) to equalize the difference in field uniformity of each projector, and also subsample the input domain of all possible colors.

\subsection{Sub-sampling in the Spatial Domain}

Instead of generating a color map $C_{p s t}$ for each pixel $(s, t)$ of each projector $p$, we propose the following simplification. Let us define a proper subset of pixels $S \subset\{(s, t) \mid 0 \leq s<w i d t h, 0 \leq t<$ height $\}$ defined by sub-sampling the projector frame-buffer based on the pattern of color variation across the projector. For every pixel $(s, t)$, the color map is a weighted average of the color maps of its nearest neighbors in $S$. We believe that the biggest problem here is to decide on the number of samples required to adequately correct the color variations across the projector FOV.

\subsection{Sub-sampling in the Input Domain}

The idea of sub-sampling the input space and generating the intermediate colors using interpolation is not new. [12, 11, 15] propose different kinds of sampling and interpolation techniques for doing this. However, none of these proposed techniques are computationally intensive and complex and cannot be implemented in real-time. The applications of large tiled displays (presented in Section 1) demands a real-time solution for the color-matching problem.

If we were doing a correction for a monochrome projector, we would generate a mapping on a domain of $2^{8}$ inputs. Since color consists of three independent channels, $r, g$ and $b$, each of which is functionally equivalent to a single monochrome channel, an immediate possibility for subsampling the input domain $I$ is to define a mapping for each of these channels independently. This reduces the input domain space cardinality from $2^{24}$ to $3 \times 2^{8}$.

However, we need to design this mapping in such a way, that the colors projected by superposition of the independent color maps for each channel, match. Mathematically, instead of finding two complete color maps as in Equation 6, we want to subsample the input domain by approximating $C$ and $C^{\prime}$ by a set of per channel color maps $C_{k}$ and $C_{k}^{\prime}, k \in\{r, g, b\}$. Thus we need to define $C_{k}$ and $C_{k}^{\prime}$ in such a way that $\forall i=\left(i_{r}, i_{g}, i_{b}\right) \in I$,

$$
\left(C_{r}\left(i_{r}\right), C_{g}\left(i_{g}\right), C_{b}\left(i_{b}\right)\right) \equiv\left(C_{r}^{\prime}\left(i_{r}\right), C_{g}^{\prime}\left(i_{g}\right), C_{b}^{\prime}\left(i_{b}\right)\right)
$$

In the next section, we describe our techniques for generating such per channel color maps.
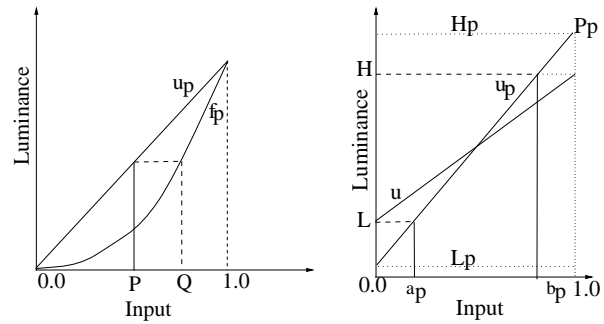

Figure 4: Left : Non-Linearity Correction; Right : Luminance Correction

\subsection{Per Channel Color Map Generation}

We describe the process of generating the mapping function for one channel. This method is repeated for the three channels - red, green and blue. The method is comprised of three steps : Non-Linearity Correction, Luminance Matching and Chrominance Matching. Each of these steps produces an intermediate mapping function. The final mapping function for each channel is a simple concatenation of the maps generated by these three steps.

\subsubsection{Non-Linearity Correction}

The input-output luminance response of each channel of a projector is found to be non-linear (Figure 3). While conventional CRT devices also exhibit non-linearities that are typically addressed by gamma correction, we allow for a more general class of nonlinearities and correct for them.

Let us denote the input to a particular channel as $i_{k}$. Let us denote the luminance corresponding to $i_{k}=0$ and the $i_{k}=1.0$ projected by a projector $p$ as $L_{p}$ and $H_{p}$ respectively. The nonlinearity correction method generates a mapping function $g$ to linearize the input-output response for the projector as shown in Figure 4. $S=f_{p}\left(i_{k}\right)$ is the piecewise linear curve generated by measuring the luminance response of projector $p$. To achieve a linear response $u_{p}\left(i_{k}\right)$, every input $i_{k}$ is mapped to an input $g_{p}\left(i_{k}\right)$ such that $g_{p}\left(i_{k}\right)=f_{p}^{-1} u_{p}\left(i_{k}\right)$. Thus the response we get after the nonlinearity correction is $f_{p}\left(g_{p}\left(i_{k}\right)\right)=f_{p}\left(f_{p}^{-1}\left(u_{p}\left(i_{k}\right)\right)\right)=u_{p}(l)=$ $L_{p}+\left(H_{p}-L_{p}\right) i_{k}$.

\subsubsection{Luminance Matching}

We choose a luminance $L$ which is the maximum of all $L_{p}$ 's (brightest black) and a luminance $H$ which is the minimum of all $H_{p}$ 's (dimmest white). Figure 4 depicts the method to generate a luminance map. The goal is to compress the luminance range of each projector within $L$ and $H$ (within the brightest black and dimmest 

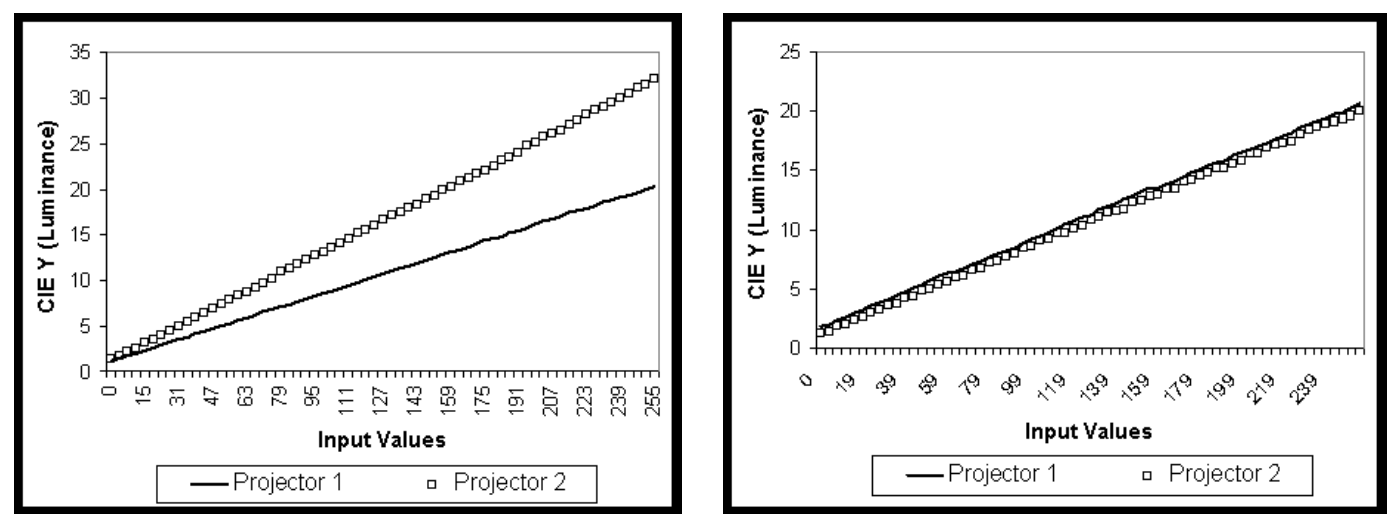

Figure 5: Luminance Response for for the Red Channel of two Projectors. Left : Before Luminance Matching : Right : After Luminance Matching. The response of the other channels are similar.

white) to achieve a response $u\left(i_{k}\right)=L+(H-L) i_{k}$. For this we generate a luminance map $B_{p}\left(i_{k}\right)=a_{p}+\left(b_{p}-a_{p}\right) i_{k}$ where $a_{p}=u_{p}^{-1}(L)$ and $b_{p}=u_{p}^{-1}(H)$. Thus, the response we get after the luminance correction is

$$
\begin{aligned}
u_{p}\left(B_{p}(l)\right) & =u_{p}\left(a_{p}+b_{p} i_{k}-a_{p} i_{k}\right) \\
& =u_{p}\left(a_{p}\right)+i_{k} u_{p}\left(b_{p}\right)-i_{k} u_{p}\left(a_{p}\right) \\
& =L+i_{k} H-i_{k} L=L+i_{k}(H-L)=u\left(i_{k}\right)
\end{aligned}
$$

Thus after non-linearity and luminance matching, every input $i_{k}$ is mapped to $g_{p}\left(B_{p}\left(i_{k}\right)\right)$. With this transformation, each channel $k$ of all projectors have exactly the same channel response $u\left(i_{k}\right)$ with the same highest and lowest luminance levels. In other words, the luminance of the response of the same input $i_{k}$ from two different projectors $p$ and $p^{\prime}$ differ by $\epsilon$. Now, since the luminance of the response for any input $i=\left(i_{r}, i_{g}, i_{b}\right) \in I$ is a summation of the luminances of the responses of each of $i_{r}, i_{g}$ and $i_{b}$, the luminance of the same input $i$ from two different projectors $p$ and $p^{\prime}$ can differ by a maximum of $3 \epsilon$. Thus, after the luminance matching step we have minimized the luminance term in the distance between the responses of the same input from different projectors which now depends entirely on the chromaticity differences.

Figure 3 shows a typical chromaticity response for our projectors ${ }^{1}$. Here we observe that for each channel $k(k \in r, g, b)$, the chromaticity coordinates $\left(x_{i_{k}}, y_{i_{k}}\right)$ of the CIE response of $i_{k}$ remain relatively constant over a large input range, $0.1 \leq i_{k} \leq 1.0$. We also observe that this constant value is nearly equivalent for different kinds of projectors. Table 1 shows the average chromaticity coordinates of the different brands of projectors and the maximum distances between the same channel of two different brand projectors.

In this scenario, the distance between two colors from different projectors, which now depends only on the chrominance terms, becomes a constant and, we cannot minimize the distance any further by just using independent per channel color maps. Since the constant chromaticity value for the response of each channel of different projectors in the input range of $0.1 \leq i_{k} \leq 1.0$ are close to each other, the luminance matching step will attain a good color match.

But, this characteristic is not true for the whole input range and the chromaticities of the different projectors differ significantly in the input range $0.0 \leq i_{k} \leq 0.1$. Thus, there is a possibility of improving the overall distance differences in this input range by relaxing the luminance match to reduce the differences in chrominance. and thus achieve a perceptually better color match.

\footnotetext{
${ }^{1}$ Four Sharp XG-E3000U, two NEC MT-1035, one nView D700Z and a Sony VPL-X600U
}

\begin{tabular}{|c||r|r|r|r|r|r|}
\hline \multicolumn{1}{|c||}{ Projector Brand } & \multicolumn{2}{c|}{ Red } & \multicolumn{2}{c|}{ Green } & \multicolumn{2}{|c|}{ Blue } \\
\cline { 2 - 7 } & $x$ & $y$ & $x$ & $y$ & $x$ & $y$ \\
\hline Sharp & 0.62 & 0.32 & 0.33 & 0.62 & 0.14 & 0.07 \\
NEC & 0.55 & 0.31 & 0.35 & 0.57 & 0.15 & 0.09 \\
nView & 0.54 & 0.34 & 0.28 & 0.58 & 0.16 & 0.07 \\
\hline Max Distance & \multicolumn{2}{|c|}{0.085} & \multicolumn{2}{c|}{0.086} & \multicolumn{2}{c|}{0.028} \\
\hline
\end{tabular}

Table 1: Chromaticity Coordinates of the primaries of different brands of projectors

\subsubsection{Chrominance Matching}

To generate a chromaticity map for each channel, we begin by identifying one of the projectors arbitrarily as the reference projector $p_{r}$. The chromaticity map of the reference projector is an identity mapping function. The chromaticity map for all other projectors is generated as follows.

for every input $i_{k}, 0.0 \leq i_{k} \leq 1.0$ in projector $p_{r}$ find the response $R\left(i_{k}\right)=\left(\bar{Y}_{i_{k}}, x_{i_{k}}, y_{i_{k}}\right)$ of $g_{p_{r}}\left(B_{p_{r}}\left(i_{k}\right)\right)$; endfor

for every input $i_{k}, 0.0 \leq i_{k} \leq 0.1$ in projector $p$

find $i_{k}^{\prime} \in\left(i_{k}-T, i_{k}+T\right)$ such that the distance

$v_{i_{k}^{\prime}}$ between $R\left(i_{k}^{\prime}\right)=\left(Y_{i_{k}^{\prime}}, x_{i_{k}^{\prime}}, y_{i_{k}^{\prime}}\right)$ and $R\left(i_{k}\right)$ is minimum Map input $i_{k}$ of projector $p$ to $i_{k}^{\prime}$

endfor

We should search for $i_{k}^{\prime}$ within the entire input space of $0.0-1.0$ so that we get the best match, but we have empirically found that $T=0.2$ is a good estimate to reduce the search space.

Now we show that after the chrominance matching step, for any input $\left(i_{r}, i_{g}, i_{b}\right) \in I$, the condition given by Equation 7 is satisfied. Let the maximum of all $v_{l^{\prime}}$ (calculated during the chrominance matching step) be $\epsilon$. Let us consider two projectors $p$ and $p^{\prime}$ and their responses for an input $C=(r, g, b)$ be $R(C)=\left(Y_{C}, x_{C}, y_{C}\right)$ and $R^{\prime}(C)=\left(Y_{C}^{\prime}, x_{C}^{\prime}, y_{C}^{\prime}\right)$ respectively. Let $R(r, 0,0)=\left(Y_{r}, x_{r}, y_{r}\right)$ and $R^{\prime}(r, 0,0)=\left(Y_{r}^{\prime}, x_{r}^{\prime}, y_{r}^{\prime}\right)$. The responses of $(0, g, 0)$ and $(0,0, b)$ are similarly defined.

By the design of the chromaticity map,

$$
\begin{aligned}
& D\left(R(r, 0,0), R^{\prime}(r, 0,0)\right) \leq \epsilon \\
& D\left(R(0, g, 0), R^{\prime}(0, g, 0)\right) \leq \epsilon \\
& D\left(R(0,0, b), R^{\prime}(0,0, b)\right) \leq \epsilon
\end{aligned}
$$

We have to prove that $D\left(R(C), R^{\prime}(C)\right)$ is bounded. The above equations and Equation 3 imply that $\forall k \in(r, g, b)$ the following 

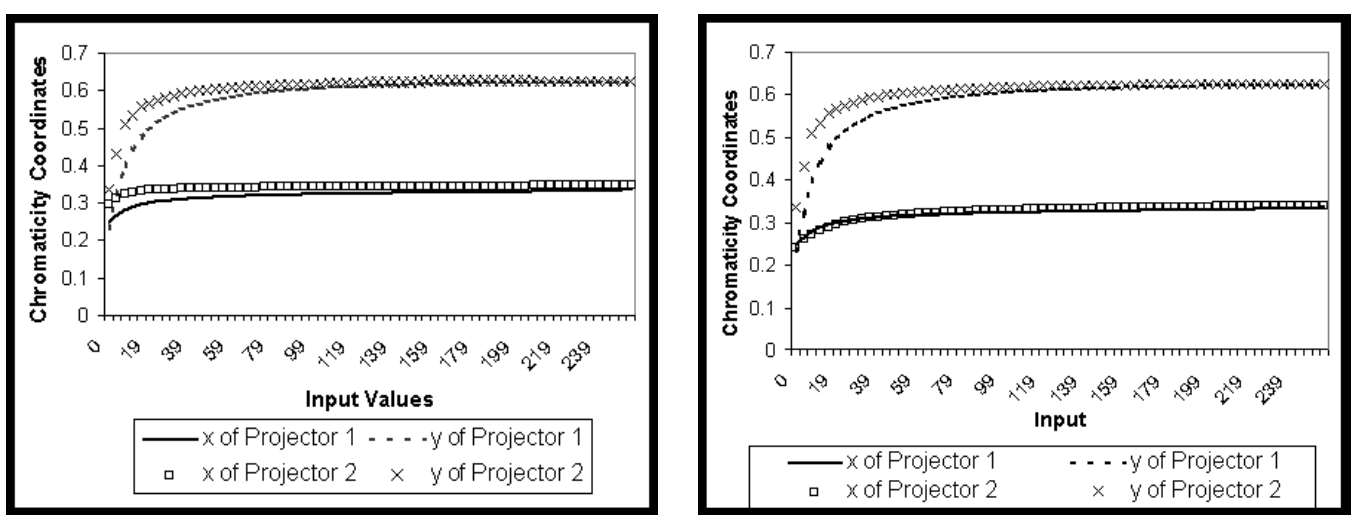

Figure 6: Chromaticity Response for the Two Projectors for the green channel. Left : Before Chrominance Matching; Right : After Chrominance Matching. The response of the other channels are similar

holds

$$
\begin{gathered}
\frac{Y_{r}-Y_{r}^{\prime}}{Y_{w}} \leq \epsilon \\
\frac{x_{r}-x_{r}^{\prime}}{1+\beta \sqrt{x_{r} x_{r}^{\prime}}} \leq \epsilon \\
\frac{y_{r}-y_{r}^{\prime}}{1+\theta \sqrt{y_{r} y_{r}^{\prime}}} \leq \epsilon
\end{gathered}
$$

Now, using Equations 1 and 2

$x_{C}-x_{C}^{\prime}=p_{r}\left(x_{r}-x_{r}^{\prime}\right)+p_{g}\left(x_{g}-x_{g}^{\prime}\right)+\left(1-p_{g}-p_{r}\right)\left(x_{b}-x_{b}^{\prime}\right)$

Thus $x_{C}-x_{C}^{\prime}$ is a convex combination of $\left(x_{k}-x_{k}^{\prime}\right), k \in(r, g, b)$. Further, $x_{k} x_{j}^{\prime} \leq 1.0$ for $k, j \in(r, g, b)$. Using these results we can show that

$$
\frac{x_{C}-x_{C}^{\prime}}{1+\beta\left(x_{C} x_{C}^{\prime}\right)^{1 / 2}} \leq \epsilon(1+\beta)
$$

Similarly,

$$
\frac{y_{C}-y_{C}^{\prime}}{1+\beta\left(y_{C} y_{C}^{\prime}\right)^{1 / 2}} \leq \epsilon(1+\theta)
$$

Also, the luminance difference

$$
\frac{1}{Y_{w}}\left(\left(Y_{r}+Y_{g}+Y_{b}\right)-\left(Y_{r}^{\prime}+Y_{g}^{\prime}+Y_{b}^{\prime}\right)\right) \leq 3 \epsilon
$$

Hence

$$
D\left(R(C), R^{\prime}(C)\right)<\sqrt{\epsilon^{2}\left((1+\beta)^{2}+(1+\theta)^{2}+9\right)}=\frac{10}{3} \epsilon
$$

\subsection{Hardware Solutions}

The solution presented in section 4.5 can be implemented in realtime using conventional hardware. Most image generators include a hardware look-up table (LUT) per channel, which is normally used for CRT gamma correction, as the last functional block before digital-to-analog conversion. We propose to use this same LUT to implement our color map. Given access to this LUT, we can replace the standard gamma correction with the color map detailed above, thereby achieving color matching of the projectors. Ideally we would like more than one color map to represent the spatial domain of each projector, as discussed in Section 4.3. But in most graphics hardware we have access to only 8-bit image channels and LUTs and cannot afford to sacrifice color intensity resolution. However. in other high-end systems with image and LUT width of $10 / 12$ bits, it will be possible to allocate $2 / 4$ bits to access different color maps associated with 4/16 different spatial region of the projector image. Our results in the color plate show that implementing the solution in Section 4.5 even for one spatial sample per projector can significantly improve color matching.

\subsection{Optical Sensing Device}

In selecting the optical sensor used in our investigation, we considered using a high quality color camera, but chose to utilize a precision spectroradiometer. By selecting the spectroradiometer, we could work in a laboratory-calibrated device-independent color space and avoid converting the measurements from the devicedependent color space of the camera. We also avoid the possibility that the camera color gamut might not completely encompass the projector color gamuts. However, it should be noted that a part of our algorithm is directed towards making it very easy to use a camera as an optical sensing device. We have designed our algorithm to do the luminance and chrominance matching separately, instead of doing it in one single step using some popular distance metric [10]. When a camera is used instead of a spectroradiometer, it does not limit the measurement of luminance. Thus the luminance matching step of our algorithm will yield similar results, when implemented using a linear response color camera.

With regard to using a camera for the chrominance matching, methods exist for converting to device independent space from the camera's color space [16] and for gamut mapping ${ }^{2}[10,17,18,19]$. Since gamut mapping is a many-to-one mapping, one will not be able to generate the 'gold standard' results produced by the spectroradiometer in the chrominance matching step, unless the color gamuts of all the projectors happen to be within the camera color gamut. However, assuming that the camera has a good gamut mapping algorithm $[10,14]$, the results of the chrominance matching step will be comparable to that achieved by the spectroradiometer.

\section{Implementation}

We have implemented our solution for display of up to four projectors, both abutting and overlapping, on a planar surface, with one color map per channel of each projector generated at only one sample pixel in the projector's FOV. Thus our solution assumes uniform color across the FOV of each individual projector (Case I.a in section 2).

We have used this method to calibrate and compare several different LCD and Texas Instruments $\mathrm{DLP}^{\mathrm{TM}}$ based projector combi-

\footnotetext{
${ }^{2}$ Gamut Mapping is a method to map a out-of-gamut color to a color in the device gamut that has the least distance to it.
} 
nations. We applied the methods to three combinations of abutted projector pairs : Two Sharp XG-E3000U , a nView D700Z and a Sony VPL-X600U, and a nView D700Z and a Sharp XG-E3000U. Lastly, we used four NEC MT-1035 projectors, overlapping each other, in three different configurations to form large tiled displays. To correct for the photometric variation in the overlapping region, we employ a blending function as described in $[6,7]$.

We used a highly diffuse, close to Lambertian material for our projector screen. We kept the settings, location and orientation of a projector the same for all the experiments during the process of matching its color, so that the results have a consistent interpretation and are repeatable. We used a Photo Research PR-705 spectroradiometer and measured the output response of each projector for 64 equally spaced input values between 0.0 and 1.0 . To demonstrate our algorithm, we use OpenGL ${ }^{\mathrm{TM}}$ rendering, where the user can load and access a color look up table. We achieved similar results for all pairs of projectors and hence present the results for the pair of Sharp projectors only.

Verification of Non-Linearity Correction : Figure 3 shows the input-output response before the non-linearity correction step. The straight line in Figure 3 shows the response of all the channels after this step.

Verification of Luminance Matching : In Figure 5 we show the luminance response curve of the red channel of two different projectors, before and after the luminance matching step. The curves are almost coincident after the luminance matching. In Figure 7 we present the luminance response for grays to show that the matching works for achromatic colors even if we have done the matching for each of the channels separately.

Verification of Chrominance Matching : In Figure 7, we show the chromaticity response of the grays after the chrominance matching step. The response is very close to $(0.33,0.33)-$ which shows that we have maintained the balance of the contribution of red, green and blue, while applying chrominance matching separately to each channel. In Figure 6, we show the chromaticity response of the red channel of two different projectors before and after the chrominance matching step. This shows that we have improved the chrominance matching while preserving the luminance matching. We also do an input-output response analysis for the projectors after the chrominance matching step and find the chromaticity coordinates of each channel are still near constant.

\section{Results}

We present the results of our algorithm in the color plates and the Quicktime ${ }^{\mathrm{TM}}$ movie in the electronic version of the proceedings. We show our results using two sets of horizontal color bars. One of these two sets of colors are chosen to have the input values in the region where the constant chromaticity characteristic is not satisfied. Our algorithm appears to achieve perceptually acceptable results in that region also. We believe that for some colors in our color plate images, the process of going from a digital camera to a color printer may have exaggerated chromaticity mismatches compared to the actual observed results.

In the color plate, we show each of the correction steps for a nView-Sony projector pair. We show the final results for a nViewSharp pair. In these two cases, we deliberately adjusted the image controls of our projectors to make them differ greatly in luminance and color to demonstrate the matching capabilities of our algorithm. But, in such cases, the brighter projector loses much of its dynamic range after calibration. Hence, we ran our color correction methods on a pair of Sharp projectors after we adjusted the brightness and the contrast control of the projectors to match the black and

\begin{tabular}{|c||r|r|r|r|}
\hline \multicolumn{1}{|c||}{ Input $(r, g, b)$} & \multicolumn{4}{c|}{$E_{94}$ color } \\
\cline { 2 - 5 } & nView-Sony pair & \multicolumn{2}{|c|}{ Sharp } \\
\cline { 2 - 5 } & Before & After & Before & After \\
\hline$(1.0,0.0,0.0)$ & 23.03 & 1.07 & 2.15 & 1.53 \\
$(0.0,1.0,0.0)$ & 221.36 & 1.46 & 31.92 & 1.18 \\
$(0.0,0.0,1.0)$ & 16.83 & 0.95 & 4.86 & 0.09 \\
$(0.0,1.0,1.0)$ & 239.31 & 2.32 & 37.60 & 0.70 \\
$(1.0,0.0,1.0)$ & 38.75 & 1.99 & 3.78 & 1.79 \\
$(1.0,1.0,0.0)$ & 244.33 & 2.86 & 30.90 & 1.70 \\
$(0.5,0.0,0.0)$ & 7.78 & 0.15 & 8.12 & 0.15 \\
$(0.0,0.5,0.0)$ & 60.46 & 3.71 & 10.92 & 0.70 \\
$(0.0,0.0,0.5)$ & 4.86 & 0.19 & 0.51 & 0.16 \\
$(0.0,0.5,0.5)$ & 63.92 & 3.72 & 12.75 & 0.53 \\
$(0.5,0.0,0.5)$ & 11.98 & 0.32 & 7.80 & 0.19 \\
$(0.5,0.5,0.0)$ & 67.07 & 3.62 & 4.63 & 1.05 \\
$(0.13,0.04,0.22)$ & 2.55 & 0.08 & 2.07 & 3.93 \\
$(0.26,0.09,0.30)$ & 5.88 & 0.48 & 4.34 & 4.75 \\
$(0.30,0.98,0.04)$ & 221.71 & 1.17 & 29.13 & 1.20 \\
$(0.43,0.87,0.11)$ & 197.22 & 3.60 & 26.10 & 0.07 \\
$(1.0,1.0,1.0)$ & 237.37 & 24.65 & 36.10 & 2.40 \\
$(0.0,0.0,0.0)$ & 0.92 & 0.17 & 0.90 & 0.37 \\
\hline Mean & 92.52 & 2.92 & 8.59 & 1.23 \\
\hline Std. Dev. & 100.30 & 5.59 & 12.00 & 1.35 \\
\hline
\end{tabular}

Table 2: Color differences before and after calibration

white levels. We used our spectroradiometer as the feedback device while doing this adjustment. Thus we preserved the dynamic range of the projectors as much as possible after calibration. We also show the results of our color calibration on large tiled displays made up of four overlapping projectors. In the electronic version of the proceedings, we show the real-time performance of our algorithm by rendering a dynamic scene using color look-up-tables. However, the digital images of the dynamic scene in the color plates illustrates the color matching better.

To quantify the amount of correction from the nView-Sony and the pair of Sharp projectors before and after photometric calibration, we show the $E_{94}$ distance between two similar inputs in a perceptually uniform CIE LUV [20] color space in Table 2.

Observations : As has been observed by many visual perceptual studies before, we also find that luminance is more important for perception than chrominance. Further, as expected by the design of our algorithm, the correction after the chrominance matching step is perceivable only for the color which have one of the inputs in the range $0.0 \leq r, g, b \leq 0.1$. Lastly, when a color from one projector is not within the color gamut of another, it is impossible to match the color exactly. In such cases, if we have overlapping projectors with some blending function to blend in the overlapping region, instead of the abutting ones, then the smooth transition makes the mismatch perceptually less noticeable.

\section{Conclusions}

Photometric uniformity across different projectors of a multiprojector display is a non-trivial problem, and we have made an attempt to solve it using the basic principles of photometry. We have proposed a repeatable method to match the colors of a system of arbitrarily different projectors using an accurate optical sensing device like a spectroradiometer. Further, we have demonstrated a practical implementation of the solution to show how it can be used to apply the photometric correction in real-time.

In the future we would like to utilize multiple color maps per projector to compensate for display field non-uniformity within a single projector. We are in the process of mounting the spectroradiometer to a pan-tilt unit to automate the measurement process of 

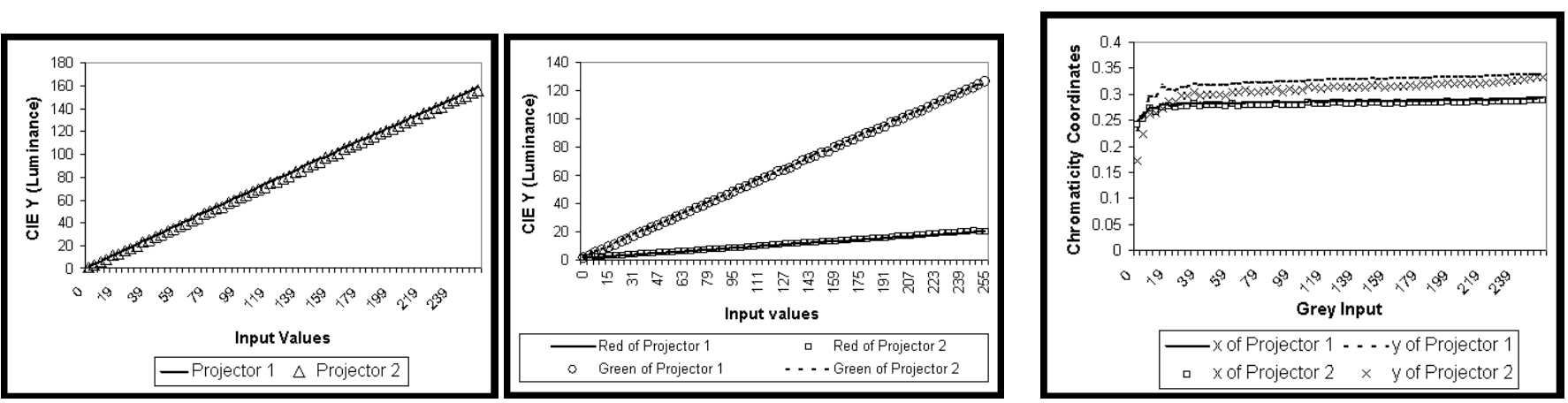

Figure 7: Left : Luminance Response for Grays after Luminance matching; Middle : Luminance Response of red and green channels after Chrominance Matching. The response for blue is similar. Right : Chrominance response of gray after chrominance matching.

the different spatial points in a projector's FOV. We can use our high end SGI with 12-bit LUTs to implement spatially indexed color map corrections. We wish to investigate the use of low-cost color cameras to replace the spectroradimeter. Further, we would like to explore projector image controls for improved luminance and color balance before applying our color map method, thereby reducing the loss of dynamic range. Lastly, though we achieved results that were perceivably acceptable (as shown in Table 5), we would like redefine the same algorithm in a perceptually uniform color space.

In conclusion, the paradigm we have presented for color matching light projectors can be applied today to improve the presentation quality of tiled displays. Further, it establishes a foundation that will enable us and other researchers to look for further improved, application-oriented solutions to this difficult problem.

\section{Acknowledgements}

This work was supported by DOE ASCI Views program; the "National Tele-Immersion Initiative (NTII)" sponsored by Advanced Network and Services, Inc. and the NSF Cooperative Agreement no. ASC-8920219 : "Science and Technology Center for Computer Graphics and Scientific Visualization"; We also thank the Intel Corporation for their generous equipment donation as a part of Technology for Education 2000.

\section{References}

[1] G.Humphreys and P.Hanrahan, "A Distributed Graphics System for Large Tiled Displays", Proceedings of IEEE Visualization '99.

[2] Cruz-Neira, Carolina, D.J.Sandin and T.A.Defanti "Surround-Screen ProjectionBased Virtual Reality : The Design and Implementation of the CAVE", ACM Computer Graphics (Proceedings of Siggraph '93).

[3] R.Samanta, J.Zheng, T.Funkhouse, K.Li and J.P.Singh, "Load Balancing for Multi-Projector Rendering Systems", SIGGRAPH/Eurographics Workshop on Graphics Hardware, August 1999 (See also http://www.cs.princeton.edu/omnimedia/index.html, cited October 20, 1999).

[4] R.Raskar, G.Welch, M.Cutts, A.Lake, L.Stesin, H.Fuchs, "The Office of the Future : A Unified Approach to Image Based Modeling and Spatially Immersive Displays", ACM Computer Graphics (Proceedings of Siggraph '98) , pp. 179188.

[5] R.Raskar, M.Cutts, G.Wech, W.Stuerzlinger, " Efficient Image Generation for Multiprojector Multisurface Displays", 9th Eurographics Rendering Workshop, '98.

[6] R.Raskar,cG.Wech, H.Fuchs, "Seamless Projection Overlaps Using Image Warping and Intensity Blending", Fourth International Conference on Virtual Systems and Multimedia, November '98.

[7] R.Raskar, M.S. Brown, R.Yang, W.Chen, G.Welch, H.Towles, B.Seales, H.Fuchs. 1999. "Multi-Projector Displays Using Camera-Based Registration," Proceedings of IEEE Visualization 99, San Fransisco, CA, October 24-29, 1999.

[8] M.Herald, I.R.Judson, R.L.Stevens, “ Introduction to Building Projector-Based Tiled Display Systems”, IEEE Computer Graphics and Applications, July/August 2000, pp 22-28.
[9] D.R.Schikore, R.A.Fischer, R.Frank, R.Gaunt, J.Hobson, B.Whitlock, "High Resolution Multiprojector Display Walls", IEEE Computer Graphics and Applications, July/August 2000, pp 38-44.

[10] N.Katoh, S.Ohno, "Three-dimensional Gamut Mapping Using Various Color Difference Formulae and Color Spaces", Journal of Electronic Imaging 8(4), October 1999, pp 365-379.

[11] J.M.Kasson, S.I.Nin, W.Plouffe, J.L.Hafner, "Performing Color Space Conversions with Three-dimensional Linear Interpolation ", Journal of Electronic Imaging 4(3), July 1995, pp 226-250.

[12] P.C. Hung, "Colorimetric Calibration in Electronic Imaging Devices Using a Look-Up-Table Model and Interpolations", Journal of Electronic Imaging 2(1), October 1993, pp 53-61.

[13] R.Balasubramanian, R. de Queiroz, Z.Fan, "Multiresolution Color Correction", SPIE Proceedings 3300, Color Imaging : Device Independent Color, Color Hardcopy and Graphics Arts III, 1998, pp 163-172.

[14] K.E.Spaulding, R.N.Ellson, J.R.Sullivan, "UltraColor : A New Gamut Mapping Strategy", SPIE Proceedings 2414, Device Independent Color Imaging II, 1998 pp 61-68.

[15] J.M.Kasson, W.Plouffe, S.I.Nin, "A Tetrahedral Interpolation Technique for Color Space Conversion”, SPIE Proceedings 1909, Device Independent Color Imaging and Imaging Systems Intergration, January 1993, pp 127-138.

[16] D.Brydges, F.Deppner, H.Kunzil, K.Heuberger, R.D.Hersch, "Application of a 3-CCD color Camera for Colorimetric and Densitometric Measurements", SPIE Proceedings 3300, Color Imaging : Device Independent Color, Color Hardcopy and Graphics Arts III, 1998, pp 292-301.

[17] N.Tsumura et. al., "Color Gamut Mapping based on Mahalanobis Distance for Color Reproduction of Electronic Endoscope Image under Different Illuminant", Proceeding of Imaging Science and Technology/SID's Color Imaging Conference, 1997, pp 158-162.

[18] N.Katoh, M.Ito, "Gamut Mapping for Computer Generated Images", Proceeding of Imaging Science and Technology/SID's Color Imaging Conference, 1996, pp 126-129.

[19] S.Nakauchi et. al., "Color Gamut Mapping by Optimizing Perceptual Image Quality", Proceeding of Imaging Science and Technology/SID's Color Imaging Conference, 1996, pp 63-67.

[20] Edited by H.John Durrett, Interactive Systems Laboratory, San Marcos, Texas, "Color and the Computer", Academic Press, INC, 1986

[21] E.J. Giorgianni, T.E. Madden, "Digital Color Management Encoding Solutions", Addison-Wesley, 1998

[22] A.Majumder, "Color Perception and Measurement", University of North Carolina at Chapel Hill, Department of Computer Science, Technical Report TR99033. 